\title{
Ambiguous Classes in the Games $\mu$-Calculus Hierarchy
}

\author{
André Arnold and Luigi Santocanale* \\ LaBRI - Université Bordeaux 1 \\ \{andre.arnold, santocan\}@labri.fr
}

\begin{abstract}
Every parity game is a combinatorial representation of a closed Boolean $\mu$-term. When interpreted in a distributive lattice every Boolean $\mu$-term is equivalent to a fixed-point free term. The alternationdepth hierarchy is therefore trivial in this case. This is not the case for non distributive lattices, as the second author has shown that the alternation-depth hierarchy is infinite.

In this paper we show that the alternation-depth hierarchy of the games $\mu$-calculus, with its interpretation in the class of all complete lattices, has a nice characterization of ambiguous classes: every parity game which is equivalent both to a game in $\Sigma_{n+1}$ and to a game in $\Pi_{n+1}$ is also equivalent to a game obtained by composing games in $\Sigma_{n}$ and $\Pi_{n}$.
\end{abstract}

\section{Introduction}

Parity games have shown to be a fundamental tool in the theory of automata recognizing infinite objects and of the logics by which these objects are usually defined 23. Among these logics we list monadic second order logic, the propositional modal $\mu$-calculus, and the collection of their fragments, i.e. logics of computation such as PDL, LTL, CTL, etc. The use of the games is not restricted to the theory but carries over to applications such as model-checking [9] or the synthesis of controllers [4].

In the monograph [3] parity games are used to establish strong relationships between $\mu$-calculi and classes of automata (on words, on trees, on Kripke structures, etc.) A class of automata is given the structure of a $\mu$-calculus by defining a composition operation $\mathcal{A}[\mathcal{B} / x]$ on automata and two fixed-point operations $\mu x . \mathcal{A}$ and $\nu x . \mathcal{A}$. Recall that a $\mu$-calculus is a set of syntactical entities with an intended functional interpretation on a complete lattice $L$, each term $t$ of arity $\operatorname{ar}(t)$ being interpreted as a monotonic mapping from $L^{\operatorname{ar}(t)}$ to $L$. The terms $\theta x . t$ of a $\mu$-calculus, for $\theta \in\{\mu, \nu\}$, are interpreted as extremal fixed-points of $t$, so that $\theta x . t$ and $t[\theta x . t / x]$ denote the same object. In the $\mu$-calculus of automata, the complete lattice $L$ is a powerset of a set of objects (words, trees, etc.); the interpretation of an automaton, as a term of empty arity of the $\mu$-calculus, coincides with the language of objects it accepts.

\footnotetext{
* The second author acknowledges financial support from the European Commission through an individual Marie Curie fellowship.
} 


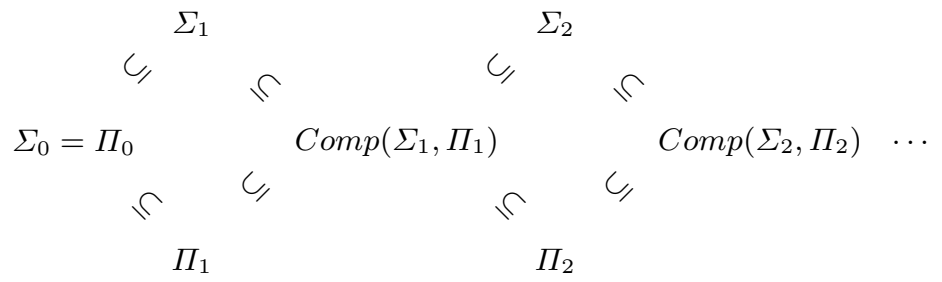

Fig. 1. The alternation-depth hierarchy

Parity games are syntactically similar to automata, thus the composition and fixed-point operations can be defined on games as well. In the monograph [3] this was not done, as, by analogy with the case of automata, the interpretation of a game is whether some distinguished position is winning or not. Since there are only two objects in the interpretation domain, such a $\mu$-calculus of games is not very interesting 11 On the other hand, for a given $\mu$-calculus, it is also possible to consider as the intended interpretation a given class of complete lattices. It turns out that considering the class of distributive (complete) lattices is not enough to make the $\mu$-calculus of games nontrivial. In this case, every term is equivalent to a term with no applications of the fixed-point operations. In order to have a $\mu$-calculus of games with a nontrivial interpretation one needs to consider the class of all complete lattices. In [20] a $\mu$-calculus with such interpretation is considered. It is defined a preorder $\leq$ on the collection $\mathcal{G}$ of games, in a constructive way. The quotient of this collection of games under the equivalence relation $\sim$ on $\mathcal{G}$ induced by $\leq$ is a lattice (although not a complete one) where the interpretation of $\theta x . G$ is indeed an extremal fixed-point. This is moreover the universal $\mu$-lattice, that is the universal lattice in which every $\mu$-term has an interpretation. By saying that this algebraic object is universal we mean that two terms $s, t$ satisfy $s \leq t$ in the quotient $\mathcal{G} / \sim$ if and only if this relation holds in every lattice where all $\mu$-terms are interpretable. Moreover the relation $s \leq t$ holds in $\mathcal{G} / \sim$ if and only if it holds in every complete lattice.

The extremal fixed-point operations of $\mu$-calculi are syntactic operators analogous to quantifiers. There have been few proposals to classify $\mu$-terms into classes according to the number of nested applications of fixed-point operations; most of these classifications happen to be equivalent [14, 15, 16]. We recall here the hierarchy of $\mu$-terms into classes proposed in [15]. The class $\Sigma_{0}=\Pi_{0}$ is the class of $\mu$-terms with no application of the fixed-point operations; $\Sigma_{n+1}$ (resp. $\left.\Pi_{n+1}\right)$ is the closure of $\Sigma_{n}$ and $\Pi_{n}$ under the composition operation and the least fixed-point operation (resp. the greatest fixed-point operation). Also, the class $\operatorname{Comp}\left(\Sigma_{n}, \Pi_{n}\right)$ is defined as the closure of $\Sigma_{n}$ and $\Pi_{n}$ under the composition operation. These classes are ordered by the inclusions as shown in figure1. As far as we are dealing with the syntax, these inclusions are obviously strict. However,

${ }^{1}$ It is also possible to say that the interpretation of a game is the set of winning strategies for a given player. This idea was pursued in [21. 
if a $\mu$-calculus comes with an intended interpretation, the relevant question is whether these inclusions are strict in the interpretation, and this question has no obvious answer. For the propositional modal $\mu$-calculus the semantical strictness of these inclusions was proved in [6, 13, 1]. For the $\mu$-calculus of parity games with its interpretation in the class of all complete lattices these inclusions were shown to be strict in 18 .

In this paper we investigate an orthogonal problem. It is easily seen that the relation

$$
\operatorname{Comp}\left(\Sigma_{n}, \Pi_{n}\right)=\Sigma_{n+1} \cap \Pi_{n+1}
$$

holds in every $\mu$-calculus, at least at the syntactic level; it can be asked whether such equality still holds with respect to a given interpretation. This question is inspired by the characterization of the ambiguous classes in the Borel hierarchy [12, $§ 11 . B, \S 22.27]$, of which, there are already known analogies within $\mu$-calculi. Indeed, it is an easy exercise to show that if a language of infinite words is accepted both by a deterministic automaton in $\Sigma_{n+1}$ and by a deterministic automaton in $\Pi_{n+1}$, then this language is accepted by a deterministic automaton in $\operatorname{Comp}\left(\Sigma_{n}, \Pi_{n}\right)$. We remark that questions related to the alternation-depth hierarchy for deterministic automata on infinite words tend to be easy, for example this hierarchy is even decidable [17]. Also, it was shown in [2] that if language of trees is definable both by an automaton in $\Sigma_{2}$ and by an automaton in $\Pi_{2}$, then it is also definable by an automaton in $\operatorname{Comp}\left(\Sigma_{1}, \Pi_{1}\right)$.

We show that, for the $\mu$-calculus of games with its interpretation in the class of all complete lattices, the equality (11) holds semantically, for every $n \geq 0$. As far as we know, together with the observation that this equality holds for languages of infinite words defined by deterministic automata, this is the first complete result of this type for $\mu$-calculi. We do not answer the analogous question for other $\mu$-calculi but we believe that the ideas and tools presented here can be adapted to other contexts. These tools are proof-theoretic in nature: the main technical proposition is an interpolation theorem 2 that we prove essentially with Maehara's method [22 $\S 1.6 .5]$. The proofs that we consider here are however circular, see [19, and being able to apply existing proof-theoretic techniques is not straightforward.

The paper is organized as follows. In section 1 we define parity games with draws and the operations on these games. We also define their semantics as monotonic mappings on a complete lattice. In section 2 we organize parity games with draws into a $\mu$-calculus and define the syntactical preorder that characterizes the semantical order relation. In section 3, we firstly recall the definition and basic facts about the hierarchy; then we prove that equality (1) holds at the semantical level.

\footnotetext{
${ }^{2}$ This interpolation result concerns the hierarchy of fixed points; it contrasts with the uniform interpolation property of the modal $\mu$-calculus 7 . which concerns the common language of two formulas and does not take into account the hierarchy of fixed points, since the main tool to prove it are disjunctive normal forms.
} 


\section{Parity Games with Draws}

A parity game with draws is a tuple $G=\left\langle\operatorname{Pos}_{E}^{G}, \operatorname{Pos}_{A}^{G}, \operatorname{Pos}_{D}^{G}, M^{G}, \rho^{G}\right\rangle$ where:

- $\operatorname{Pos}_{E}^{G}, \operatorname{Pos}_{A}^{G}, \operatorname{Pos}_{D}^{G}$ are finite pairwise disjoint sets of positions (Eva's positions, Adam's positions, and draw positions),

$-M^{G}$, the set of moves, is a subset of $\left(\operatorname{Pos}_{E}^{G} \cup \operatorname{Pos}_{A}^{G}\right) \times\left(\operatorname{Pos}_{E}^{G} \cup \operatorname{Pos}_{A}^{G} \cup \operatorname{Pos}_{D}^{G}\right)$,

$-\rho^{G}$ is a mapping from $\left(\operatorname{Pos}_{E}^{G} \cup \operatorname{Pos}_{A}^{G}\right)$ to $\mathbb{N}$.

Whenever an initial position is specified, these data define a game between player Eva and player Adam. The outcome of a finite play is determined according to the normal play condition: a player who cannot move loses. It can also be a draw, if a position in $\operatorname{Pos}_{D}^{G}$ is reached 3 The outcome of an infinite play $\left\{\left(g_{k}, g_{k+1}\right) \in\right.$ $\left.M^{G}\right\}_{k \geq 0}$ is determined by means of the rank function $\rho^{G}$ as follows: it is a win for Eva if and only if the number

$$
\max \left\{i \in \mathbb{N} \mid \exists \text { infinitely many } k \text { s.t. } \rho^{G}\left(g_{k}\right)=i\right\}
$$

is even. To simplify the notation, we shall use $\operatorname{Pos}_{E, A}^{G}$ for the set $\operatorname{Pos}_{E}^{G} \cup \operatorname{Pos}_{A}^{G}$ and use similar notations such as $\operatorname{Pos}_{E, D}^{G}$, etc. We let $\operatorname{Max}{ }^{G}=\max \rho^{G}\left(\operatorname{Pos}_{E, A}^{G}\right)$ if the set $\operatorname{Pos}_{E, A}^{G}$ is not empty, and $M a x^{G}=-1$ otherwise.

\subsection{Operations on Parity Games}

We define here some operations and constants on games. When defining operations on games we shall always assume that the sets of positions of distinct games are pairwise disjoint.

Meets and Joins. For any finite set $I, \bigwedge_{I}$ is the game defined by letting $\operatorname{Pos}_{E}=\emptyset, \operatorname{Pos}_{A}=\left\{p_{0}\right\}, \operatorname{Pos}_{D}=I, M=\left\{\left(p_{0}, i\right) \mid i \in I\right\}$ (where $\left.p_{0} \notin I\right)$, $\rho\left(p_{0}\right)=0$. The game $\bigvee_{I}$ is defined similarly, exchanging $\operatorname{Pos}_{E}$ and $\operatorname{Pos}_{A}$.

Composition Operation. Given two games $G$ and $H$ and a mapping $\psi$ : $P_{D}^{G} \longrightarrow P_{E, A, D}^{H}$, the game $K=G \circ_{\psi} H$ is defined as follows:

$-\operatorname{Pos}_{E}^{K}=\operatorname{Pos}_{E}^{G} \cup \operatorname{Pos}_{E}^{H}$,

$-\operatorname{Pos}_{A}^{K}=\operatorname{Pos}_{A}^{G} \cup \operatorname{Pos}_{A}^{H}$,

$-\operatorname{Pos}_{D}^{K}=\operatorname{Pos}_{D}^{H}$,

$-M^{K}=\left(M^{G} \cap\left(\operatorname{Pos}_{E, A}^{G} \times \operatorname{Pos}_{E, A}^{G}\right)\right) \cup M^{H}$

$\cup\left\{\left(p, \psi\left(p^{\prime}\right)\right) \mid\left(p, p^{\prime}\right) \in M^{G} \cap\left(\operatorname{Pos}_{E, A}^{G} \times \operatorname{Pos}_{D}^{G}\right)\right\}$.

- $\rho^{K}$ is such that its restrictions to the positions of $G$ and $H$ are respectively equal to $\rho^{G}$ and $\rho^{H}$.

Sum Operation. Given a finite collection of parity games $G_{i}, i \in I$, their sum $H=\sum_{i \in I} G_{i}$ is defined in the obvious way:

- $P_{Z}^{H}=\bigcup_{i \in I} P_{Z}^{G_{i}}$, for $Z \in\{E, A, D\}$,

$-M^{H}=\bigcup_{i \in I} M^{G_{i}}$,

- $\rho^{H}$ is such that its restriction to the positions of each $G_{i}$ is equal to $\rho_{i}^{G}$.

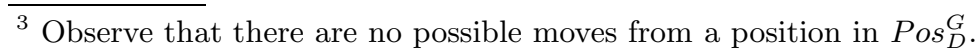


Fixed-point Operations. If $G$ is a game, a system on $G$ is a tuple $S=$ $\langle E, A, M\rangle$ where:

- $E$ and $A$ are pairwise disjoint subsets of $\operatorname{Pos}_{D}^{G}$,

- $M \subseteq(E \cup A) \times \operatorname{Pos}_{E, A, D}^{G}$.

Given a system $S$ and $\theta \in\{\mu, \nu\}$, we define the parity game $\theta_{S} \cdot G$ :

$-\operatorname{Pos}_{E}^{\theta_{S}} \cdot G=\operatorname{Pos}_{E}^{G} \cup E$,

$-\operatorname{Pos}_{A}^{\theta_{S}} \cdot G=\operatorname{Pos}_{A}^{G} \cup A$,

$-\operatorname{Pos}_{D}^{\theta_{S} \cdot G}=\operatorname{Pos}_{D}^{G}-(E \cup A)$,

- $M^{\theta_{S} \cdot G}=M^{G} \cup M$,

- $\rho^{\theta_{S} \cdot G}$ is the extension of $\rho^{G}$ to $E \cup A$ such that:

- if $\theta=\mu$, then $\rho^{\theta_{S}} \cdot G$ takes on $E \cup A$ the constant value $M a x^{G}$ if this number is odd or $\operatorname{Max}^{G}+1$ if $\operatorname{Max}^{G}$ is even,

- if $\theta=\nu$, then $\rho^{\theta_{S}} \cdot G$ takes on $E \cup A$ the constant value $M a x^{G}$ if this number is even or $\operatorname{Max}^{G}+1$ if $\operatorname{Max}^{G}$ is odd.

Predecessor Game. Let $G$ be a game such that $M a x^{G} \neq-1$, i.e. there is at least one position in $\operatorname{Pos}_{E, A}^{G}$. Let $\operatorname{Top}^{G}=\left\{g \in \operatorname{Pos}_{E, A}^{G} \mid \rho^{G}(g)=\operatorname{Max}^{G}\right\}$, then the predecessor game $G^{-}$is defined as follows:

$-\operatorname{Pos}_{E}^{G^{-}}=\operatorname{Pos}_{E}^{G}-\operatorname{Top}^{G}$,

$-\operatorname{Pos}_{A}^{G^{-}}=\operatorname{Pos}_{A}^{G}-\operatorname{Top}^{G}$,

$-\operatorname{Pos}_{D}^{G^{-}}=\operatorname{Pos}_{D}^{G} \cup \operatorname{Top}^{G}$,

$-M^{G^{-}}=M^{G}-\left(\operatorname{Top}^{G} \times \operatorname{Pos}_{E, A, D}^{G}\right)$,

$-\rho^{G^{-}}$is the restriction of $\rho^{G}$ to $\operatorname{Pos}_{E, A}^{G^{-}}$.

\subsection{Semantics of Parity Games}

Given a complete lattice $L$, the intepretation of a parity game $G$ is going to be a monotone mapping of the form

$$
\|G\|: L^{P_{D}^{G}} \longrightarrow L^{P_{E, A}^{G}}
$$

where $L^{P_{K}^{G}}$ is the $P_{K}^{G}$-fold product lattice of $L$ with itself. If $g \in \operatorname{Pos}_{A, E}^{G}$ then $\left\|G_{g}\right\|$ will denote the projection of $\|G\|$ onto the $g$ coordinate. Any parity game $G$ can be reconstructed in a unique way from the predecessor game $G^{-}$by one application of some fixed-point operation $\theta_{S}$; moreover the predecessor game is "simpler". Thus we define the interpretation of a parity game inductively. If $P_{E, A}^{G}=\emptyset$, then $L^{P_{E, A}^{G}}=L^{\emptyset}=1$, the complete lattices with just one element, and there is just one possible definition of the mapping $\|G\|$. Otherwise, if $\operatorname{Max}^{G}$ is odd, then $\|G\|$ is the parameterized least fixed-point of the monotone mapping

$$
L^{P_{E}^{G}, A} \times L^{P_{D}^{G}} \longrightarrow L^{P_{E, A}^{G}}
$$


defined by the system of equations:

$$
x_{g}= \begin{cases}\bigvee\left\{x_{g^{\prime}} \mid\left(g, g^{\prime}\right) \in M^{G}\right\} & \text { if } g \in \operatorname{Pos}_{E}^{G} \cap \operatorname{Top}^{G} \\ \bigwedge\left\{x_{g^{\prime}} \mid\left(g, g^{\prime}\right) \in M^{G}\right\} & \text { if } g \in \operatorname{Pos}_{A}^{G} \cap \operatorname{Top}^{G} \\ \left\|G_{g}^{-}\right\|\left(X_{T_{o p}}, X_{\operatorname{Pos}_{D}^{G}}\right) & \text { otherwise. }\end{cases}
$$

If $M a x^{G}$ is even, then $\|G\|$ is the parameterized greatest fixed-point of this mapping.

\section{The $\mu$-Calculus of Games}

Let $X$ be a countable set of variables. A pointed parity game with labeled draws is a tuple $\left\langle G, p_{\star}^{G}, \lambda^{G}\right\rangle$ where $G$ is a parity game, $p_{\star}^{G} \in \operatorname{Pos}_{E, A, D}^{G}$ is a specified initial position, and $\lambda^{G}: \operatorname{Pos}_{D}^{G} \longrightarrow X$ is a labeling of draw positions by variables. With $\mathcal{G}$ we shall denote the collection of all pointed parity games with labeled draws; as no confusion will arise, we will call a pointed parity game with labeled draws simply "game". Similarly, we shall abuse the notation and write $G$ to denote the entire tuple $\left\langle G, p_{\star}^{G}, \lambda^{G}\right\rangle$. With the notation $G_{g}$ we shall denote the game that differs from $G$ only in that the initial position is now $g$, i.e $p_{\star}^{G_{g}}=g$.

We give the collection $\mathcal{G}$ the structure of a $\mu$-calculus, as defined in [3, $\S 2.1]$. If $x$ is a variable, the game $\hat{x}$ has just one draw position labeled by $x$. The arity of a game $G$, denoted by $\operatorname{ar}(G)$, is the subset of variables $\lambda^{G}\left(\operatorname{Pos}_{D}^{G}\right)$.

A substitution is a mapping $\sigma: X \longrightarrow \mathcal{G}$; given a game $G$ and a substitution $\sigma$, the composition of $G$ and $\sigma$-for which we use the notation $G[\sigma]$ - is defined as

$$
G[\sigma]=\left(G \circ_{\psi} \sum_{x \in \operatorname{ar}(G)} \sigma(x)\right),
$$

where $\psi(g)=p_{\star}^{\sigma\left(\lambda^{G}(g)\right)}$ for $g \in \operatorname{Pos}_{D}^{G}$. Moreover, $p_{\star}^{G[\sigma]}=p_{\star}^{G}$ and $\lambda^{G[\sigma]}(p)=$ $\lambda^{\sigma(x)}(p)$ whenever $p \in \operatorname{Pos}_{D}^{\sigma(x)}$. Therefore $\operatorname{ar}(G[\sigma])=\bigcup_{x \in \operatorname{ar}(G)} \operatorname{ar}(\sigma(x))$.

Similarly, given $G$ in $\mathcal{G}$ and $x \in X$, let $\operatorname{Pos}_{x}=\left\{g \in \operatorname{Pos}_{D}^{G} \mid \lambda^{G}(g)=x\right\}$. Define the system $S$ as $\left\langle\emptyset, \operatorname{Pos}_{x}, \operatorname{Pos}_{x} \times\left\{p_{\star}^{G}\right\}\right\rangle$. Then we define

$$
\theta x \cdot G=\left(\theta_{S} \cdot G\right),
$$

where moreover $\lambda^{\theta x . G}$ is the restriction of $\lambda^{G}$ and $p_{\star}^{\theta x . G}=p_{\star}^{G}$. Remark that $\theta x . G=G$ if $x \notin \operatorname{ar}(G)$.

The above constructions are analogous to those given in $[3, \S 7.2$ ] for automata and therefore one can mimic the proof presented there to show that $\mathcal{G}$ with this structure satisfies the axioms of a $\mu$-calculus.

Observe that the operation of forming the predecessor game $G^{-}$can be extended to pointed parity games with labeled draws if we choose a variable $x_{g} \notin \operatorname{ar}(G)$ for each $g \in \operatorname{Top}^{G}$ : we let in this case $\lambda^{G^{-}}$be the extension of $\lambda^{G}$ such that $\lambda^{G^{-}}(g)=x_{g}$ for $g \in T o p^{G}$. 


\subsection{The Preorder on $\mathcal{G}$}

In order to describe a preorder on the class $\mathcal{G}$, we shall define a new game $\langle\langle G, H\rangle\rangle$ for a pair of games $G$ and $H$ in $\mathcal{G}$. This is not a pointed parity game with draws as defined in the previous section; to emphasize this fact, the two players will be named Mediator and Opponent instead of Eva and Adam.

Before formally defining the game $\langle\langle G, H\rangle\rangle$, we give its informal description and explanation. Mediator's goal is to prove that the relation $\|G\| \leq\|H\|$ holds in any complete lattice; Opponent's goal is to show that this relation does not hold. For example, if $G=\bigvee_{i \in I} G_{i}$ has the shape of a join and $H=\bigwedge_{j \in J} H_{j}$ has the shape of a meet, then this is an Opponent's position: Mediator should be prepared to prove $\left\|G_{i}\right\| \leq\left\|H_{j}\right\|$ for any pair of indexes $i$ and $j$; thus Opponent should find a pair of indexes $(i, j)$ and show that $\left\|G_{i}\right\| \not \leq\left\|H_{j}\right\|$. If $G=\bigwedge_{i \in I} G_{i}$ is a meet and $H=\bigvee_{j \in J} H_{j}$ is a join, then this is a Mediator's position: Mediator should find either an $i$ and show that $\left\|G_{i}\right\| \leq\|H\|$ or a $j$ and show that $\|G\| \leq$ $\left\|H_{j}\right\|$; Opponent should be prepared to disprove any such relation 4

Thus the game is played on the two boards, simultaneously. At a first approximation, a position of $\langle\langle G, H\rangle\rangle$ is a pair of positions from $G$ and $H$. Since we code meets as Adam's positions and joins as Eva's positions, Mediator is playing with Adam on $G$ and with Eva on $H$; Opponent is playing with Eva on $G$ and with Adam on $H$. Thus a pair $(g, h)$ in $\operatorname{Pos}_{A}^{G} \times \operatorname{Pos}_{E}^{H}$ clearly belongs to Mediator and a pair $(g, h)$ in $\operatorname{Pos}_{E}^{G} \times \operatorname{Pos}_{A}^{H}$ clearly belongs to Opponent. Pairs in $\operatorname{Pos}_{E}^{G} \times \operatorname{Pos}_{E}^{H}$ or $\operatorname{Pos}_{A}^{G} \times \operatorname{Pos}_{A}^{H}$ are ambiguous, as both players could play. The situation is not symmetric, however, as Opponent is obliged to play while Mediator is allowed to play, if he wants, but he can also decide to delay his move. In the formal definition, we code the fact that two players can play from the same pair by duplicating every pair into a Mediator's position and into an Opponent's position.

Definition 2.1. The game $\langle\langle G, H\rangle\rangle$ is defined as follows:

- The set of Mediator's positions is

$$
\operatorname{Pos}_{E, A, D}^{G} \times\{M\} \times \operatorname{Pos}_{E, A, D}^{H},
$$

and the set of Opponent's positions is

$$
\operatorname{Pos}_{E, A, D}^{G} \times\{O\} \times \operatorname{Pos}_{E, A, D}^{H} .
$$

- We describe the moves 5 by cases:

- If $(g, h) \in\left(\operatorname{Pos}_{E}^{G} \times \operatorname{Pos}_{A, D}^{H}\right) \cup\left(\operatorname{Pos}_{E, D}^{G} \times \operatorname{Pos}_{A}^{H}\right)$, then there is just one "silent" move

$$
(g, M, h) \rightarrow(g, O, h)
$$

\footnotetext{
${ }^{4}$ These moves suffice to Mediator to reach his goal, as the relation $\leq$ that we shall define turns out to be transitive. This fact is analogous to a cut-elimination theorem and to Whitman's conditions characterizing free lattices [10].

${ }^{5}$ As we wish to distinguish moves coming from $G$ and moves coming from $H$, the underlying graph of this game can have distinct edges relating the same pair of vertices.
} 
and moves of the form

$$
(g, O, h) \rightarrow\left(g^{\prime}, M, h\right) \quad(g, O, h) \rightarrow\left(g, M, h^{\prime}\right)
$$

for every move $\left(g, g^{\prime}\right) \in M^{G}$ and every move $\left(h, h^{\prime}\right) \in M^{H}$.

- If $(g, h) \in\left(\operatorname{Pos}_{A}^{G} \times \operatorname{Pos}_{E, D}^{H}\right) \cup\left(\operatorname{Pos}_{A, D}^{G} \times \operatorname{Pos}_{E}^{H}\right)$, then there is just one silent move

$$
(g, O, h) \rightarrow(g, M, h)
$$

and moves of the form

$$
(g, M, h) \rightarrow\left(g^{\prime}, O, h\right) \quad(g, M, h) \rightarrow\left(g, O, h^{\prime}\right)
$$

for every move $\left(g, g^{\prime}\right) \in M^{G}$ and every move $\left(h, h^{\prime}\right) \in M^{H}$.

- If $(g, h) \in\left(\operatorname{Pos}_{E}^{G} \times \operatorname{Pos}_{E}^{H}\right)$ then there are moves of the form

$$
(g, O, h) \rightarrow\left(g^{\prime}, M, h\right) \quad(g, M, h) \rightarrow\left(g, O, h^{\prime}\right)
$$

for every move $\left(g, g^{\prime}\right) \in M^{G}$ and every move $\left(h, h^{\prime}\right) \in M^{H}$, and moreover a silent move

$$
(g, M, h) \rightarrow(g, O, h) .
$$

- Similarly, if $(g, h) \in\left(\operatorname{Pos}_{A}^{G} \times \operatorname{Pos}_{A}^{H}\right)$ then there are moves of the form

$$
(g, M, h) \rightarrow\left(g^{\prime}, O, h\right) \quad(g, O, h) \rightarrow\left(g, M, h^{\prime}\right)
$$

for every move $\left(g, g^{\prime}\right) \in M^{G}$ and every move $\left(h, h^{\prime}\right) \in M^{H}$, and moreover a silent move

$$
(g, M, h) \rightarrow(g, O, h) .
$$

- Finally, if $(g, h) \in \operatorname{Pos}_{D}^{G} \times \operatorname{Pos}_{D}^{H}$, then: If $\lambda^{G}(g)=\lambda^{H}(h)$, then there is a move

$$
(g, M, h) \rightarrow(g, O, h)
$$

and no move from $(g, O, h)$. Hence this is a winning position for Mediator. If $\lambda^{G}(g) \neq \lambda^{H}(h)$, then there is a move

$$
(g, O, h) \rightarrow(g, M, h)
$$

and no move from $(g, M, h)$. The latter is a win for Opponent.

- Now let us define the winning plays for Mediator in this game. As usual a maximal finite play is lost by the player who cannot move. For infinite plays, observe that any (maximal) play $\gamma$ in $\langle\langle G, H\rangle\rangle$ defines two plays (not necessarily maximal) $\pi_{G}(\gamma)$ in $G$ and $\pi_{H}(\gamma)$ in $H$. Generalizing what happens for finite plays we say that Mediator wins an infinite play $\gamma$ if and only if either $\pi_{G}(\gamma)$ is a win for Adam on $G$, or $\pi_{H}(\gamma)$ is a win for Eva on $H$. 
In the above definition we must explain the meaning of statements such as " $\pi_{H}(\gamma)$ is a win for Eva on $H$ " whenever $\pi_{H}(\gamma)$ is a finite play which is not maximal. In this case, the last position of the play $\pi_{H}(\gamma)$ belongs either to $\operatorname{Pos}_{E}^{H}$ or to $\operatorname{Pos}_{A}^{H}$ : we say that this is a win for Adam in the first case and a win for Eva in the latter case, with the intuition that the player who gives up playing loses.

This convention allows Mediator to play just on one board and to give up on the other if Adam has a winning strategy on $G$ or Eva has a winning strategy on $H$. On the other hand, as soon as Opponent gives up on one board, he's going to lose. Notice that the game $\langle\langle G, H\rangle\rangle$ alternates between Opponent's positions and Mediator's positions, thus if a player among Mediator and Opponent gives up on one board, this is indeed his own responsibility.

Finally observe that the condition $(1)$ : " $\pi_{G}(\gamma)$ is a win for Adam on $G$, or $\pi_{H}(\gamma)$ is a win for Eva on $H$ " implies but is not equivalent to $(2)$ : "if $\pi_{G}(\gamma)$ is a win for Eva on $G$, then $\pi_{H}(\gamma)$ is a win for Eva on $H$ ". The logic is complicated by the fact that $\pi_{G}(\gamma)$ could be a draw, but this is also the only obstacle to obtain the equivalence between (1) and (2).

Definition 2.2. If $G$ and $H$ belong to $\mathcal{G}$, then we declare that $G \leq H$ if and only if Mediator has a winning strategy in the game $\langle\langle G, H\rangle\rangle$ starting from position $\left(p_{\star}^{G}, O, p_{\star}^{H}\right)$.

In the following, we shall write $G \sim H$ to mean that $G \leq H$ and $H \leq G$. We continue by listing some useful facts about the game $\langle\langle G, H\rangle$ and the relation $\leq$

Lemma 2.3. In the game $\langle\langle G, H\rangle\rangle$ Mediator has a winning strategy from a position of the form $(g, O, h)$ if and only if he has a winning strategy from $(g, M, h)$.

We do not include a proof of this lemma for lack of space.

An homomorphism from a game $G$ to a game $H$ is a mapping $f$ from the positions of $G$ to the positions of $H$ such that:

$-f\left(p_{\star}^{G}\right)=h_{\star}^{H}$,

- if $g$ belongs to $\operatorname{Pos}_{E}^{G}$ (resp. $\operatorname{Pos}_{A}^{G}$ ) then $f(g)$ belongs to $\operatorname{Pos}_{E}^{H}$ (resp. $\operatorname{Pos}_{A}^{H}$ ) and $\rho^{G}(g)=\rho^{H}(f(g))$,

- if $g$ belongs to $\operatorname{Pos}_{D}^{G}$ then $f(g)$ belongs to $\operatorname{Pos}_{D}^{H}$ and $\lambda^{G}(g)=\lambda^{H}(f(g))$,

- if $\left(g, g^{\prime}\right) \in M^{G}$ then $\left(f(g), f\left(g^{\prime}\right)\right) \in M^{H}$.

An homomorphism $f$ from a game $G$ to a game $H$ is a bisimulation if moreover:

- for any position $g$ of $G$, if $(f(g), h) \in M^{H}$ then there exists a position $g^{\prime}$ of $G$ such that $\left(g, g^{\prime}\right) \in M^{G}$ and $h=f\left(g^{\prime}\right)$.

Lemma 2.4. If there is a bisimulation from $G$ to $H$, then $G \sim H$. 
The proof of this lemma, which we do not include for lack of space, amounts to observe that both in the game $\langle\langle G, H\rangle\rangle$ and the game $\langle\langle H, G\rangle\rangle$ Mediator can use a "copycat" strategy. We can use Lemma 2.4 to establish several equivalences. Let $G$ be a game and $T \subseteq \operatorname{Pos}_{E}^{G}, A$ be a collection of positions of $G$. Let $X_{T} \subseteq X$ be a subset of variables in bijection with $T$ and such that $X_{T} \cap \operatorname{ar}(G)=\emptyset$. The game $G^{T \rightarrow X_{T}}$ is obtained as follows: every position $t \in T$ is added to the set of draw positions and labeled by the variable $x_{t}$ which corresponds to $t$. Of course there are no more moves from a position $t \in T$ in the game $G^{T \rightarrow X_{T}}$. The relation $G_{g} \sim G_{g}^{T \rightarrow X_{T}}\left[G_{t} / x_{t}\right]_{t \in T}$ holds, as a consequence of the fact that there is a bisimulation from $G_{g}^{T \leftrightarrow} X_{T}\left[G_{t} / x_{t}\right]$ to $G_{g}$. Also, let $G_{g}^{\prime}$ be the game obtained from $G_{g}$ by considering the reachable part from $g$. Again, we have $G_{g} \sim G_{g}^{\prime}$ as the inclusion of the positions of $G_{g}^{\prime}$ into the positions of $G_{g}$ is a bisimulation. Thus we are allowed to consider only games in $\mathcal{G}$ that are reachable from the initial position.

Proposition 2.5. The relation $\leq$ has the following properties:

1. It is reflexive and transitive.

2. Composition is monotonic: If $G \leq H$ and if for all $x \in X, \sigma(x) \leq \sigma^{\prime}(x)$ then $G[\sigma] \leq H\left[\sigma^{\prime}\right]$.

3. For any game $G$ and any substitution $\sigma, G \leq \bigwedge_{I}[\sigma]$ if and only if $G \leq \sigma\left(x_{i}\right)$ for all $i \in I$.

4. For any game $H$ and any substitution $\sigma, \bigvee_{I}[\sigma] \leq H$ if and only if $\sigma\left(x_{i}\right) \leq H$ for all $i \in I$.

5. For $\theta \in\{\mu, \nu\}, \theta x . G \sim G[\theta x . G / x]$.

6. If $G[H / x] \leq H$ then $\mu x . G \leq H$.

7. If $G \leq G[H / x]$ then $G \leq \nu x$. $H$.

8. It is the least relation on $\mathcal{G}$ having properties 1 to 7 .

9. It is sound and complete with respect to the class of all complete lattices: $G \leq H$ if and only if for any complete lattice $L$ and any $v: X \longrightarrow L$

$$
\left\|G_{p_{\star}^{G}}\right\|\left(v \circ \lambda^{G}\right) \leq\left\|H_{p_{\star}^{H}}\right\|\left(v \circ \lambda^{H}\right) .
$$

Proof. These properties were stated and proved in [20] for a restricted class of fair games and for a different relation $\preceq$ (similar to the one of [5, 11]). However, we can prove the following: 1$)$ the relation $\leq$ is indeed reflexive, transitive, and monotonic, 2) every game in $\mathcal{G}$ is $\leq$-equivalent to a fair game, 3 ) if $G$ and $H$ are fair games, then $G \leq H$ if and only if $G \preceq H$.

Therefore the quotient of the class of fair games under the equivalence relation induced by $\preceq$ is order isomorphic to the quotient of $\mathcal{G}$ under the equivalence relation $\sim$ and this quotient inherits all the properties proved in [20].

In particular the quotient $\mathcal{G} / \sim$ is a lattice where the greatest lower bound (resp. least upper bound) of the equivalence classes of $G_{1}, \ldots, G_{k}$ is the equivalence class of $\bigwedge_{k}\left(G_{1}, \ldots, G_{k}\right)$ (resp. $\left.\bigvee_{k}\left(G_{1}, \ldots, G_{k}\right)\right)$. It is a $\mu$-lattice as well, meaning that all the $\mu$-terms constructible from the signature $\langle T, \wedge, \perp, \vee\rangle$ are interpretable as infima, suprema, least prefixed points and greatest postfixed 
points of previously defined operations. The $\mu$-lattice $\mathcal{G} / \sim$ is freely generated by the set $X$, meaning that given any $\mu$-lattice $L$ and any mapping $\psi: X \longrightarrow L$, there exists a unique extension of $\psi$ to a mapping $\psi^{\prime}: \mathcal{G} / \sim \longrightarrow L$ that preserves the interpretation of $\mu$-terms. From this property it readily follows that $\leq$ is the least preorder having the properties listed above.

\section{Ambiguous Classes in the Mostowski Hierarchy}

If $G$ is a game then two mappings $\rho$ and $\rho^{\prime}$ from $\operatorname{Pos}_{E, A}^{G}$ to $\mathbb{N}$ are said to be equivalent with respect to $G$ if any infinite path in $G$ is winning according to $\rho$ if and only if it is winning according to $\rho^{\prime}$. Let $G$ be a game and $\rho$ be a mapping equivalent to $\rho^{G}$ w.r.t. $G$. It is easily observed that the game $G^{\prime}$ obtained from $G$ by substituting the rank function $\rho$ with $\rho^{G}$ is equivalent to $G$ : $G \sim G^{\prime}$.

Definition 3.1. We say that a game $G$ belongs to $\Sigma_{0}=\Pi_{0}$ if and only if it is acyclic. For $n \geq 1$, we say that a game $G$ belongs to $\Sigma_{n}\left(\right.$ resp. $\left.\Pi_{n}\right)$ if there is a mapping $\rho$ equivalent to $\rho^{G}$ w.r.t. $G$, and an odd (resp. even) number $m \geq n-1$ such that $\rho\left(\operatorname{Pos}_{E, A}^{G}\right) \subseteq\{m-n+1, \ldots, m\}$. We say that a game belongs to $\operatorname{Comp}\left(\Sigma_{n}, \Pi_{n}\right)$ if it can be obtained from games in $\Sigma_{n}$ and $\Pi_{n}$ by a sequence of applications of the composition operation.

Observe that, by construction, for every $n \geq 1$, if $G$ belongs to $\Sigma_{n}$ (resp. $\Pi_{n}$ ) then $\mu x . G$ belongs to $\Sigma_{n}\left(\operatorname{resp} . \Pi_{n+1}\right)$ and $\nu x . G$ belongs to $\Sigma_{n+1}\left(\operatorname{resp} . \Pi_{n}\right)$. Moreover, $\operatorname{Comp}\left(\Sigma_{0}, \Pi_{0}\right)=\Sigma_{0}$ and in general $\operatorname{Comp}\left(\Sigma_{n}, \Pi_{n}\right) \subseteq \Sigma_{n+1} \cap \Pi_{n+1}$. We shall show that the converse holds as well.

Lemma 3.2. If a game $G$ belongs to $\Sigma_{1} \cap \Pi_{1}$ then it is acyclic.

Proof. As $G$ belongs to $\Sigma_{1} \cap \Pi_{1}$ there are two mappings $\rho$ and $\rho^{\prime}$ equivalent to $\rho^{G}$ w.r.t. $G$ whose images are respectively $\{m\}$ and $\left\{m^{\prime}\right\}$, where $m$ is odd and $m^{\prime}$ is even. If $G$ is not acyclic, there exists a position $p$ in $G$ and a non empty path $\gamma$ from $p$ to $p$. The infinite path $\gamma^{\omega}$ is a win for Adam, according to $\rho$, and a win for Eva, according to $\rho^{\prime}$. This is a contradiction.

Lemma 3.3. If a game $G$ is strongly connected and belongs to $\Sigma_{n+1} \cap \Pi_{n+1}$ then either it belongs to $\Sigma_{n}$, or it belongs to $\Pi_{n}$.

Proof. If $G$ belongs to $\Sigma_{n+1} \cap \Pi_{n+1}$ then there exist two mappings $\rho$ and $\rho^{\prime}$, equivalent to $\rho^{G}$ w.r.t. $G$, whose images are respectively included in $\{m-$ $n, \ldots, m\}$ and $\left\{m^{\prime}-n, \ldots, m^{\prime}\right\}$ where $m$ is odd and $m^{\prime}$ is even.

Assume that there are two positions $p, p^{\prime} \in \operatorname{Pos}_{E, A}^{G}$ such that $\rho(p)=m$ and $\rho^{\prime}\left(p^{\prime}\right)=m^{\prime}$. Since $G$ is strongly connected, there exists a non empty path $\gamma$ from $p$ to $p^{\prime}$ and a non empty path $\gamma^{\prime}$ from $p^{\prime}$ to $p$. The maximal value of $\rho$ (resp. $\rho^{\prime}$ ) which occurs infinitely often in the path $\left(\gamma \gamma^{\prime}\right)^{\omega}$ is $m$ (resp. $\left.m^{\prime}\right)$. Therefore this infinite path is a win for Adam according to $\rho$ and a win for Eva according to $\rho^{\prime}$, a contradiction as $\rho$ and $\rho^{\prime}$ are equivalent. 
It follows that either $\rho$ never takes the value $m$ on $\operatorname{Pos}_{E, A}^{G}$ or $\rho^{\prime}$ never takes the value $m^{\prime}$ on $\operatorname{Pos}_{E, A}^{G}$. In the first case $\rho\left(\operatorname{Pos}_{E, A}^{G}\right) \subseteq\{m-n, \ldots, m-1\}$ and $G \in \Pi_{n}$. In the second case $\rho^{\prime}\left(\operatorname{Pos}_{E, A}^{G}\right) \subseteq\left\{m^{\prime}-n, \ldots, m^{\prime}-1\right\}$ and $G \in \Sigma_{n}$.

Corollary 3.4. If a game $G$ belongs to $\Sigma_{n+1}$ and to $\Pi_{n+1}$, then it belongs to $\operatorname{Comp}\left(\Sigma_{n}, \Pi_{n}\right)$.

Proof. If $n=0$ then this is lemma 3.2. Otherwise we can construct $G$ from its maximal strongly connected components $G_{i}$ by means of a sequences of substitutions. According to lemma 3.3 , each of the $G_{i}$ is either in $\Sigma_{n}$ or in $\Pi_{n}$. Therefore $G \in \operatorname{Comp}\left(\Sigma_{n}, \Pi_{n}\right)$.

Thus we have argued that the equality (1) holds at the syntactic level. In the introduction we have stressed that the relevant question is whether such equality holds with respect to the given interpretation of all complete lattices. By the characterization in [20], this is the same as asking whether such equation holds up to the equivalence relation $\sim$ induced by the preorder $\leq$.

Definition 3.5. Let $G \in \mathcal{G}$ and say that $G \in \mathcal{S}_{n}$ if there exists a $G^{\prime} \in \Sigma_{n}$ such that $G \sim G^{\prime}$. Similarly, say that $G \in \mathcal{P}_{n}$ if there exists a $G^{\prime} \in \Pi_{n}$ such that $G \sim G^{\prime}$, and that $G \in \mathcal{C}_{n}$ if there exists a $G^{\prime} \in \operatorname{Comp}\left(\Sigma_{n}, \Pi_{n}\right)$ such that $G \sim G^{\prime}$.

The ambiguous class $\mathcal{D}_{n}$ is simply the intersection of $\mathcal{P}_{n}$ and $\mathcal{S}_{n}$. The main result of this paper is the following:

Theorem 3.6. The ambiguous class $\mathcal{D}_{n+1}=\mathcal{P}_{n+1} \cap \mathcal{S}_{n+1}$ and the class $\mathcal{C}_{n}$ are equal, for every $n \geq 0$.

The relation $\mathcal{C}_{n} \subseteq \mathcal{P}_{n+1} \cap \mathcal{S}_{n+1}$ immediately follows from the definition of the classes $\mathcal{C}_{n}, \mathcal{S}_{n+1}, \mathcal{P}_{n+1}$ and by the relation (1). For the converse it is enough to prove the following Proposition.

Proposition 3.7. Let $G$ and $H$ be games in $\Pi_{n+1}$ and $\Sigma_{n+1}$, respectively, and suppose that $G \leq H$. Then there exists a $K \in \operatorname{Comp}\left(\Sigma_{n}, \Pi_{n}\right)$ such that $G \leq K$ and $K \leq H$.

Indeed, if $G^{\prime} \in \mathcal{S}_{n+1} \cap \mathcal{P}_{n+1}$, then let $G \in \Pi_{n+1}$ and $H \in \Sigma_{n+1}$, such that $G^{\prime} \sim G \sim H$. If $K$ is as in the statement of Proposition 3.7, then the relations

$$
G^{\prime} \leq G \leq K \leq H \leq G^{\prime}
$$

exhibit $G^{\prime}$ as a member of $\mathcal{C}_{n}$.

Proof (of Proposition 3.7). Let us fix $G \in \Pi_{n+1}$ and $H \in \Sigma_{n+1}$, thus we shall assume that $\rho^{G}\left(\operatorname{Pos}_{E, A}^{G}\right) \subseteq\{m-n, \ldots, m\}$ where $m$ is even and that $\rho^{H}\left(\operatorname{Pos}_{E, A}^{H}\right) \subseteq\left\{m^{\prime}-n, \ldots, m^{\prime}\right\}$ with $m^{\prime}$ odd. We also assume that $G \leq H$ and fix a winning strategy for Mediator in the game $\langle\langle G, H\rangle\rangle$ from position $\left(p_{\star}^{G}, O, p_{\star}^{H}\right)$. This game is almost 6 a game whose set of infinite winning plays is described by

\footnotetext{
${ }^{6}$ The winning condition can be described using Rabin pairs on the edges.
} 
a Rabin acceptance condition. Thus, if Mediator has a winning strategy in this game, then he has a deterministic bounded memory winning strategy as well. Therefore we shall assume that the fixed winning strategy is deterministic and has a bounded memory. We shall represent it as the tuple $\left\langle S, U, s_{\star}, \psi\right\rangle$, where $\left\langle S, U, s_{\star}\right\rangle$ is a finite pointed graph, with set of memory states $S$, set of update transitions $U$, and an initial state $s_{\star} ; \psi$ is an homomorphism of graphs from $\left\langle S, U, s_{\star}\right\rangle$ to the graph of $\langle\langle G, H\rangle\rangle$ (mapping every memory state to a position and an update transition to a move) with the following properties:

$-\psi\left(s_{\star}\right)=\left(p_{\star}^{G}, O, p_{\star}^{H}\right)$,

- if $s \in S$ and $\psi(s)=(g, O, h)$ is an Opponent's position, then for every move $(g, O, h) \rightarrow\left(g^{\prime}, M, h^{\prime}\right)$ there exists a unique $s^{\prime}$ such that $s \rightarrow s^{\prime}$ and $\psi\left(s^{\prime}\right)=\left(g^{\prime}, M, h^{\prime}\right)$,

- if $s \in S$ and $\psi(s)=(g, M, h)$ is a Mediator's position, then there exists a unique transition $s \rightarrow s^{\prime}$,

- if $s_{0} \rightarrow s_{1} \rightarrow \ldots$ is an infinite path in the graph $\langle S, U\rangle$, then the infinite play $\psi\left(s_{0}\right) \rightarrow \psi\left(s_{1}\right) \rightarrow \ldots$ is a win for Mediator.

Recall from 1.1 the definition of the predecessor game $G^{-}$. In particular, recall that $\operatorname{Top}^{G}=\left\{g \in \operatorname{Pos}_{E, A}^{G} \mid \rho^{G}(g)=\operatorname{Max}^{G}\right\}$ and that $\operatorname{Top}^{H}=\{h \in$ $\left.\operatorname{Pos}_{E, A}^{H} \mid \rho^{H}(h)=M a x^{H}\right\}$. Observe that, for the games $G$ and $H$ under consideration, $G^{-}$belongs to $\Sigma_{n}$ and $H^{-}$belongs to $\Pi_{n}$. Intuitively, our next goal is to show that we can completely decompose the given winning strategy into a collection of local strategies that Mediator can play either in $\left\langle\left\langle G, H^{-}\right\rangle\right\rangle$, or in $\left\langle\left\langle G, H^{\prime}\right\rangle\right\rangle$ for some game $H^{\prime}$ of the form $\bigwedge_{I}$, or in $\left\langle\left\langle G^{-}, H\right\rangle\right\rangle$ or in some $\left\langle\left\langle G^{\prime}, H\right\rangle\right\rangle$ for some game $G^{\prime}$ of the form $\bigvee_{I}$.

We shall denote by $[s]$ the maximal strongly connected component of the graph $\langle S, U\rangle$ to which $s$ belongs. We observe that the following exhaustive and exclusive cases arise:

(Ac) The component $[s]$ is reduced to the singleton $\{s\}$. Observe that we cannot have a transition $s \rightarrow s$ as the graph of $\langle\langle G, H\rangle\rangle$ is bipartite. Therefore the component $[s]$ is acyclic.

(Cy) The component $[s]$ is cyclic (and contains at least two elements). We have the following subcases:

(CyA) The projection of $[s]$ onto $H$ is stuck and belongs to Adam: let $s_{1}, s_{2} \in[s]$ be such that $s_{1} \rightarrow s_{2}$ and let $\psi\left(s_{i}\right)=\left(g_{i}, P_{i}, h_{i}\right)$ for $i=1,2$; then $h_{1}=h_{2} \in \operatorname{Pos}_{A}^{H}$ and the move $\left(g_{1}, P_{1}, h_{1}\right) \rightarrow\left(g_{2}, P_{2}, h_{1}\right)$ is either a left move (i.e. $\left.\left(g_{1}, g_{2}\right) \in M^{G}\right)$ or it is a silent move.

(CyE) The projection of $[s]$ onto $G$ is stuck and belongs to Eva: the formal definition is obtained by exchanging $H$ with $G$ and Adam with Eva in the definition of ( $\mathrm{CyA})$.

The previous conditions do not hold and:

(CyG) The projection of $[s]$ onto $G$ contains a visit to $T o p^{G}$ : there exists an $s^{\prime} \in[s]$ such that $\psi\left(s^{\prime}\right)=\left(g^{\prime}, P^{\prime}, h^{\prime}\right)$ and $\rho^{G}\left(g^{\prime}\right)=\operatorname{Max}{ }^{G}$.

(CyH) The projection of $[s]$ onto $H$ contains a visit to $\operatorname{Top}^{H}$ : there exists an $s^{\prime} \in[s]$ such that $\psi\left(s^{\prime}\right)=\left(g^{\prime}, P^{\prime}, h^{\prime}\right)$ and $\rho^{H}\left(h^{\prime}\right)=M a x^{H}$. 
(CyN) None of the previous conditions hold. In particular, for all $s^{\prime} \in$ $[s]$, if $\psi\left(s^{\prime}\right)=\left(g^{\prime}, P^{\prime}, h^{\prime}\right), g^{\prime} \in \operatorname{Pos}_{E, A}^{G}$ implies $\rho^{G}\left(g^{\prime}\right)<\operatorname{Max}{ }^{G}$ and $h^{\prime} \in \operatorname{Pos}_{E, A}^{H}$ implies $\rho^{G}\left(h^{\prime}\right)<\operatorname{Max}^{H}$.

The reader should verify that the above cases are indeed disjoint. To see that $(C y A)$ and $(C y E)$ are disjoint, observe that a proper cycle in the graph of $\langle\langle G, H\rangle\rangle$ cannot be stuck both on $G$ and on $H$. To see that $(C y G)$ and $(C y H)$ are disjoint consider a maximal strongly connected component $[s]$ that visits both $T o p^{G}$ and $T o p^{H}$, and a path $\gamma$ that visits all the states in $[s]$. The unique way the path $\psi\left(\gamma^{\omega}\right)$ can be a win in the game $\langle\langle G, H\rangle\rangle$ for Mediator is that the play $\psi(\gamma)$ is stuck on $H$ on an Adam's position, in which case [ $s]$ satisfies $(C y A)$, or that this play is stuck on $G$ on an Eva's position, in which case $[s]$ satisfies $(C y E)$.

Definition 3.8. We say that $s \mapsto s^{\prime}$ if and only if there exists a path $s=s_{0} \rightarrow$ $s_{1} \rightarrow \ldots \rightarrow s_{n}=s^{\prime}$, but $s^{\prime}$ does not belong to the strongly connected component of $s$.

Clearly, the relation $s \mapsto s^{\prime}$ is irreflexive and acyclic, and therefore well founded.

Lemma 3.9. Let $s \in S$ and $\psi(s)=(g, P, h)$, where $P \in\{O, M\}$. Suppose that the strongly connected component $[s]$ is of type $(C y G)$ or of type $(C y N)$. If for each $h^{\prime} \in$ Top $^{H}$ there exists $\kappa\left(h^{\prime}\right)$ such that $G_{g^{\prime}} \leq \kappa\left(h^{\prime}\right)$ whenever $s \mapsto s^{\prime}$ and $\psi\left(s^{\prime}\right)=\left(g^{\prime}, P^{\prime}, h^{\prime}\right)$, then

$$
G_{g} \leq H_{h}^{-}\left[\kappa\left(h^{\prime}\right) / y_{h^{\prime}}\right]_{h^{\prime} \in T o p^{H}}
$$

Of course there is a dual lemma if the stronlgy connected component $[s]$ is of type $(\mathrm{CyH})$; we leave the reader to formulate it. Observe that in order to form a collection $\left\{\kappa\left(h^{\prime}\right)\right\}$ satisfying the hypothesis of the lemma, it is enough to let $\kappa\left(h^{\prime}\right)=\bigvee_{\emptyset}$ if there is no $s^{\prime}$ such that $s \mapsto s^{\prime}$ and $\psi\left(s^{\prime}\right)=\left(g^{\prime}, P^{\prime}, h^{\prime}\right)$.

Proof (of lemma [3.9). The positions of the game $\left\langle\left\langle G, H_{h}^{-}\left[\kappa\left(h^{\prime}\right) / y_{h^{\prime}}\right]_{h^{\prime} \in T o p^{H}}\right\rangle\right.$ form a set which is the disjoint union of a component $\operatorname{Pos}_{E, A, D}^{G} \times\{M, O\} \times$ $\left(\operatorname{Pos}_{E, A, D}^{H}-\operatorname{Top}^{H}\right)$ and of components $\operatorname{Pos}_{E, A, D}^{G} \times\{M, O\} \times \operatorname{Pos}_{E, A, D}^{\kappa\left(h^{\prime}\right)}$ for $h^{\prime} \in$ $T o p^{H}$. Moreover, in the latter components, the game is exactly as in $\left\langle\left\langle G, \kappa\left(h^{\prime}\right)\right\rangle\right\rangle$.

Mediator can use the strategy $S$ from position $\psi(s)$ on the first component $\operatorname{Pos}_{E, A, D}^{G} \times\{M, O\} \times\left(\operatorname{Pos}_{E, A, D}^{H}-\right.$ Top $\left.^{H}\right)$, as long as the strategy does not suggest a move $\left(g^{\prime}, P, h\right) \rightarrow\left(g^{\prime}, P^{\prime}, h^{\prime}\right)$ for some $h^{\prime} \in T o p^{H}$. If this is the case and if $s^{\prime}$ is the state of the strategy that lifts $\left(g^{\prime}, P^{\prime}, h^{\prime}\right)$, then $s \mapsto s^{\prime}$, because $[s]$ cannot contain a visit to $T o p^{H}$. Hence, by assumption, there is a winning strategy in the game $\left\langle\left\langle G_{g^{\prime}}, \kappa\left(h^{\prime}\right)\right\rangle\right.$ from both positions $\left(g^{\prime}, O, p_{\star}^{\kappa\left(h^{\prime}\right)}\right)$ and $\left(g^{\prime}, M, p_{\star}^{\kappa\left(h^{\prime}\right)}\right)$, by lemma 2.3. The move $\left(g^{\prime}, P, h\right) \rightarrow\left(g^{\prime}, P^{\prime}, h^{\prime}\right)$ becomes a move to $\left(g^{\prime}, P^{\prime}, p_{\star}^{\kappa\left(h^{\prime}\right)}\right)$ in $\left\langle\left\langle G, \kappa\left(h^{\prime}\right)\right\rangle\right\rangle$ and Mediator can continue with a winning strategy from the latter position. 
We complete now the proof of Proposition 3.7 by proving the following stronger claim:

Claim. For each $s \in S$ such that $\psi(s)=(g, P, h)$ there is a game $K_{s}$ in the class $\operatorname{Comp}\left(\Sigma_{n}, \Pi_{n}\right)$ such that $G_{g} \leq K_{s} \leq H_{h}$.

The proof is by induction on the well founded relation $\mapsto$ and it is subdivided into cases, according to the type of the strongly connected component $[s]$.

We suppose first that the type of $[s]$ is (Ac), so that if $s \rightarrow s^{\prime}$ then $s \mapsto s^{\prime}$. Observe that if $s \rightarrow s^{\prime}$ is a transition lifting a silent move of the form

$$
(g, O, h) \rightarrow(g, M, h) \quad(g, M, h) \rightarrow(g, O, h)
$$

then there is essentially nothing to prove: we can let $K_{s}=K_{s^{\prime}}$ since by the induction hypothesis $G_{g} \leq K_{s^{\prime}} \leq H_{h}$.

If $g \in \operatorname{Pos}_{E}^{G}$ and $P=O$, then for each move $\left(g, g^{\prime}\right) \in M^{G}$ there is a move $(g, O, h) \rightarrow\left(g^{\prime}, M, h\right)$ and a lifting $s \rightarrow s\left(g^{\prime}\right)$ of this move. By the induction hypothesis there are $K_{s\left(g^{\prime}\right)} \in \operatorname{Comp}\left(\Sigma_{n}, \Pi_{n}\right)$ such that $G_{g^{\prime}} \leq K_{s\left(g^{\prime}\right)} \leq H_{h}$. We can let $K_{s}=\bigvee_{\left(g, g^{\prime}\right) \in M^{G}} K_{s\left(g^{\prime}\right)} \in \operatorname{Comp}\left(\Sigma_{n}, \Pi_{n}\right)$, it follows that

$$
G_{g} \sim \bigvee_{\left(g, g^{\prime}\right) \in M^{G}} G_{g^{\prime}} \leq \bigvee_{\left(g, g^{\prime}\right) \in M^{G}} K_{s\left(g^{\prime}\right)} \leq H_{h}
$$

Assume now that $g \in \operatorname{Pos}_{A}^{G}, P=M$, and that the unique transition $s \rightarrow s^{\prime}$ of the strategy is suggesting a move of the form $(g, M, h) \rightarrow\left(g^{\prime}, M, h\right)$ for some $\left(g, g^{\prime}\right) \in M^{G}$. We let $K_{s}=K_{s^{\prime}} \in \operatorname{Comp}\left(\Sigma_{n}, \Pi_{n}\right)$, and knowing that $G_{g^{\prime}} \leq$ $K_{s^{\prime}} \leq H_{h}$ we derive

$$
G_{g} \sim \bigwedge_{\left(g, g^{\prime}\right) \in M^{G}} G_{g^{\prime}} \leq G_{g^{\prime}} \leq K_{s} \leq H_{h}
$$

We can use a dual argument if $h \in \operatorname{Pos}_{A}^{H}$ and $P=O$ or if $h \in \operatorname{Pos}_{E}^{H}$ and $P=M$. If $g \in \operatorname{Pos}_{D}^{G}$ and $h \in \operatorname{Pos}_{D}^{H}$, then we let $K_{s}$ be the game with only one position labeled by $\lambda^{G}(g)$.

We suppose now that the type of $[s]$ is (CyA). Observe that if $s^{\prime} \in[s]$ and $\psi\left(s^{\prime}\right)=\left(g^{\prime}, O, h\right)$ is an Opponent position, then for each move $\left(h, h^{\prime}\right) \in M^{H}$ there is a move $\left(g^{\prime}, O, h\right) \rightarrow\left(g^{\prime}, M, h^{\prime}\right)$ in $\langle\langle G, H\rangle\rangle$ and a lifting of this move $s^{\prime} \rightarrow s^{\prime}\left(h^{\prime}\right)$ in $\langle S, U\rangle$. By definition of the type (CyA), $s^{\prime}\left(h^{\prime}\right) \notin[s]$, hence there exists a $K_{s^{\prime}\left(h^{\prime}\right)}$ such that $G_{g^{\prime}} \leq K_{s^{\prime}\left(h^{\prime}\right)} \leq H_{h^{\prime}}$. We can let $K_{s^{\prime}}=\bigwedge_{\left(h, h^{\prime}\right) \in M^{H}} K_{s^{\prime}\left(h^{\prime}\right)}$ since this game belongs to $\operatorname{Comp}\left(\Sigma_{n}, \Pi_{n}\right)$ and

$$
G_{g^{\prime}} \leq \bigwedge_{\left(h, h^{\prime}\right) \in M^{H}} K_{s^{\prime}\left(h^{\prime}\right)} \leq \bigwedge_{\left(h, h^{\prime}\right) \in M^{H}} H_{h^{\prime}} \sim H_{h}
$$

If $s^{\prime} \in[s]$ and $\psi\left(s^{\prime}\right)=\left(g^{\prime}, M, h\right)$, then there is a unique transition $s^{\prime} \rightarrow s^{\prime \prime}$. If $s \mapsto s^{\prime}$ then we can use the inductive hypothesis; otherwise, if $s^{\prime \prime} \in[s]$, we observe that $\psi\left(s^{\prime \prime}\right)=\left(g^{\prime \prime}, O, h\right)$ is an Opponent position and that we have described how to construct $K_{s^{\prime \prime}}$ satisfying the claim in the previous parargraph. As the relation $G_{g^{\prime}} \leq G_{g^{\prime \prime}}$ holds, we can let $K_{s^{\prime}}=K_{s^{\prime \prime}}$, since

$$
G_{g^{\prime}} \leq G_{g^{\prime \prime}} \leq K_{s^{\prime \prime}} \leq H_{h}
$$


We suppose now that the type of $[s]$ is either $(\mathbf{C y G})$ or $(\mathbf{C y H})$. For each $h^{\prime} \in \operatorname{Top}^{H}$ let

$$
\kappa\left(h^{\prime}\right)=\bigvee_{s} K_{s^{\prime}}
$$

where the $K_{s^{\prime}} \in \operatorname{Comp}\left(\Sigma_{n}, \Pi_{n}\right)$ have been previously constructed and satisfy the relation $G_{g^{\prime}} \leq K_{s^{\prime}} \leq H_{h^{\prime}}$. Observe that $G_{g^{\prime}} \leq \kappa\left(h^{\prime}\right)$ whenever $s \mapsto s^{\prime}$ and $\psi\left(s^{\prime}\right)=\left(g^{\prime}, P^{\prime}, h^{\prime}\right)$, therefore by lemma 3.9 the relation

$$
G_{g} \leq H_{h}^{-}\left[\kappa\left(h^{\prime}\right) / y_{h^{\prime}}\right]_{h^{\prime} \in T o p^{H}}
$$

holds. Also, we have $\kappa\left(h^{\prime}\right) \leq H_{h^{\prime}}$ for all $h^{\prime} \in T o p^{H}$ and therefore

$$
H_{h}^{-}\left[\kappa\left(h^{\prime}\right) / y_{h^{\prime}}\right]_{h^{\prime} \in T o p^{H}} \leq H_{h}^{-}\left[H_{h^{\prime}} / y_{h^{\prime}}\right]_{h^{\prime} \in T o p^{H}}
$$

where the last game is clearly equivalent to $H_{h}$. If we let $K_{s}$ be the game $H_{h}^{-}\left[\kappa\left(h^{\prime}\right) / y_{h^{\prime}}\right]_{h^{\prime} \in T o p^{H}}$, then $K_{s}$ belongs to $\operatorname{Comp}\left(\Sigma_{n}, \Pi_{n}\right)$, since $H_{h}^{-} \in \Pi_{n}$, $\Pi_{n} \subseteq \operatorname{Comp}\left(\Sigma_{n}, \Pi_{n}\right)$, and for all $h^{\prime} \in \operatorname{Top}^{H} \kappa\left(h^{\prime}\right) \in \operatorname{Comp}\left(\Sigma_{n}, \Pi_{n}\right)$. Moreover we have shown that $G_{g} \leq K_{s} \leq H_{h}$.

We can use dual arguments if the strongly connected component is of type $(\mathbf{C y E})$ or $(\mathbf{C y H})$; therefore the claim holds for every $s \in S$ and for $s_{\star} \in S$ in particular. As we have $\psi\left(s_{\star}\right)=\left(p_{\star}^{G}, O, p_{\star}^{H}\right)$, the relations

$$
G=G_{p_{\star}^{G}} \leq K_{s_{\star}} \leq H_{p_{\star}^{H}}=H
$$

prove Proposition 3.7 .

Finally we remark that if there exists a bounded memory winning strategy in the game $\langle\langle G, H\rangle\rangle$ for Mediator, then there exists a winning strategy for Mediator of size $|G| \times|H|$, where $|G|=\operatorname{card} \operatorname{Pos}_{E, A, D}^{G}+\operatorname{card} M^{G}$ is the size of a game $G$. This follows from considerations developed in [8]. Thus effective bounds to construct $K$ such that $G \leq K \leq H$ can be extracted out of this information.

\section{References}

[1] A. Arnold. The $\mu$-calculus alternation-depth hierarchy is strict on binary trees. Theor. Inform. Appl., 33(4-5):329-339, 1999.

[2] A. Arnold and D. Niwiński. Fixed point characterization of weak monadic logic definable sets of trees. In Tree automata and languages (Le Touquet, 1990), volume 10 of Stud. Comput. Sci. Artificial Intelligence, pages 159-188. NorthHolland, Amsterdam, 1992.

[3] A. Arnold and D. Niwiński. Rudiments of $\mu$-calculus, volume 146 of Studies in Logic and the Foundations of Mathematics. North-Holland Publishing Co., Amsterdam, 2001.

[4] A. Arnold, A. Vincent, and I. Walukiewicz. Games for synthesis of controllers with partial information. To appear in Theoret. Comput. Sci., 2002. 
[5] A. Blass. A game semantics for linear logic. Ann. Pure Appl. Logic, 56(1-3):183$220,1992$.

[6] J. C. Bradfield. The modal mu-calculus alternation hierarchy is strict. Theoret. Comput. Sci., 195(2):133-153, 1998.

[7] G. D'Agostino and M. Hollenberg. Logical questions concerning the $\mu$-calculus: interpolation, Lyndon and łoś-Tarski. J. Symbolic Logic, 65(1):310-332, 2000.

[8] M. Dziembowski, S. Jurdziński and I. Walukiewicz. How much memory is needed to win infinite games? In Twelth Annual IEEE Symposium on Logic in Computer Science, pages 99-110. IEEE Computer Society Press, 1997.

[9] E. A. Emerson, C. S. Jutla, and A. P. Sistla. On model checking for the $\mu$-calculus and its fragments. Theoret. Comput. Sci., 258(1-2):491-522, 2001.

[10] R. Freese, J. Ježek, and J. B. Nation. Free lattices. American Mathematical Society, Providence, RI, 1995.

[11] A. Joyal. Free lattices, communication and money games. In Logic and scientific methods (Florence, 1995), pages 29-68. Kluwer Acad. Publ., Dordrecht, 1997.

[12] A. S. Kechris. Classical descriptive set theory, volume 156 of Graduate Texts in Mathematics. Springer-Verlag, New York, 1995.

[13] G. Lenzi. A hierarchy theorem for the $\mu$-calculus. In Automata, languages and programming (Paderborn, 1996), volume 1099 of Lecture Notes in Comput. Sci., pages 87-97. Springer, Berlin, 1996.

[14] A. W. Mostowski. Regular expressions for infinite trees and a standard form of automata. In Computation theory (Zaborów, 1984), volume 208 of Lect. Notes in Comput. Sci., pages 157-168. Springer, Berlin, 1985.

[15] D. Niwinski. On fixed-point clones. In Automata, languages and programming, volume 226 of Lecture Notes in Comput. Sci., pages 464-473, 1986.

[16] D. Niwiński. Fixed point characterization of infinite behavior of finite-state systems. Theoret. Comput. Sci., 189(1-2):1-69, 1997.

[17] D. Niwiński and I. Walukiewicz. Relating hierarchies of word and tree automata. In STACS 98 (Paris, 1998), volume 1373 of Lecture Notes in Comput. Sci., pages 320-331. Springer, Berlin, 1998.

[18] L. Santocanale. The alternation hierarchy for the theory of $\mu$-lattices. Theory and Applications of Categories, 9:166-197, January 2002.

[19] L. Santocanale. A calculus of circular proofs and its categorical semantics. In FOSSACS02, volume 2303 of Lecture Notes in Comput. Sci., pages 357-371, 2002.

[20] L. Santocanale. Free $\mu$-lattices. J. Pure Appl. Algebra, 168(2-3):227-264, 2002.

[21] L. Santocanale. Parity games and $\mu$-bicomplete categories. Theor. Inform. Appl., (36):195-227, 2002.

[22] G. Takeuti. Proof theory. North-Holland Publishing Co., Amsterdam, 1975. Studies in Logic and the Foundations of Mathematics, Vol. 81.

[23] W. Thomas. Languages, automata, and logic. In Rozenberg G. and Salomaa A., editors, Handbook of Formal Language Theory, volume III, pages 389-455. Springer-Verlag, New York, 1996. 